\title{
Physiological responses to heat stress on a tropical shore: the benefits of mushrooming behaviour in the limpet Cellana grata
}

\author{
Gray A. Williams ${ }^{1, *}$, Maurizio De Pirro ${ }^{2}$, Kenneth M. Y. Leung ${ }^{3,4}$, David Morritt ${ }^{3}$ \\ ${ }^{1}$ The Swire Institute of Marine Science, Department of Ecology \& Biodiversity, The University of Hong Kong, \\ Pokfulam Road, Hong Kong SAR, PR China \\ ${ }^{2}$ Dipartimento di Biologia Animale e Genetica, Università degli Studi di Firenze, 50125 Firenze, Italy \\ ${ }^{3}$ School of Biological Sciences, Royal Holloway, University of London, Egham, TW20 0EX, UK \\ ${ }^{4}$ Present address: The Swire Institute of Marine Science, Department of Ecology \& Biodiversity, \\ The University of Hong Kong, Pokfulam Road, Hong Kong SAR, PR China
}

\begin{abstract}
Sub-lethal responses to heat stress were investigated in the limpet Cellana grata. During summer low tides, foot temperatures were hotter than rock temperatures, but positively correlated with, heart rate, air and rock temperatures. Hotter limpets showed mushrooming behaviour, raising their shell from the rock. Over $30 \%$ of monitored limpets were not relocated during the subsequent daytime low tide. Missing animals were mostly situated on horizontal surfaces, were smaller than those recaptured, and had higher body temperatures, mushrooming heights and heart rates. A laboratory protocol was designed to resemble on-shore thermal stress conditions. Firstly, small and large limpets were held on a hot plate for $60 \mathrm{~min}$ and either constrained or allowed to mushroom. Secondly, unconstrained, large animals were held on the hot plate for $120 \mathrm{~min}$. Unconstrained limpets were able to mushroom and had lower foot temperatures but higher heart rates than those constrained, suggesting mushrooming is an active response. Small animals had higher heart rates than large individuals. Mantle water could not be collected from most small, mushrooming limpets but was from constrained animals, and was more concentrated in small limpets. Small and constrained limpets had more concentrated haemolymph than large or mushrooming animals. Mantle and haemolymph osmolalities were positively related, except at high mantle water osmolalities. Smaller animals lost relatively more water, and constrained limpets more than those allowed to mushroom. Large limpets on the hot plate for 120 min showed similar mushrooming heights and heart rates but had hotter foot temperatures, higher haemolymph concentrations, lost all their mantle water and nearly twice as much water than those held for $60 \mathrm{~min}$. Mushrooming behaviour appears to be a short-term, high-risk strategy that allows limpets temporary relief from stressful conditions and may increase their chance of survival until the next tidal immersion.
\end{abstract}

KEY WORDS: Heart rate $\cdot$ Heat stress · Hong Kong · Osmoregulation · Summer die-off · Tropical rocky shore

\section{INTRODUCTION}

Physical stress, such as high temperatures and desiccation, during emersion periods has long been appreciated as a causal factor in the distribution and abundance of rocky shore organisms (Connell 1961, Foster 1961, Underwood 1985, Helmuth \& Hofmann 2001).
Calm, hot summer days on temperate and tropical rocky shores can induce severe heat stress in both sessile and mobile species, often resulting in what are commonly called 'high shore kills' (e.g. algae, Hawkins \& Hartnoll 1985; barnacles, Foster 1961; and mobile species, Branch 1981, Garrity 1984, McMahon 2001). Many authors have documented stress in mobile 
molluscs in terms of elevated body temperatures and water loss (Vermeij 1971, Smith 1975, Verderber et al. 1983, Lowell 1984) or indeed mortality as a result of heat stress (Frank 1965, Wolcott 1973, Kohn 1993, Williams 1994).

In an extensive study on mobile species in the Bay of Panama, Garrity (1984) demonstrated a series of responses in these species which appeared to have adaptive benefits, such as (1) being only active whilst awash, (2) aggregation of individuals and (3) raising the shell from the rock surface to aid evaporative cooling. Garrity was able to demonstrate the benefits of such behaviours, in terms of alleviating water loss and high body temperatures, and ultimately increasing their survival value, by preventing animals from performing them. He did not, however, isolate the underlying physiological causes of these benefits. McMahon et al. (1991) specifically measured physiological changes (body temperature, heart rate and haemolymph chemistry) in the chiton Chiton stokesii during emersion periods under laboratory conditions. They concluded that a combination of physiological and behavioural adaptations allowed the chiton to tolerate 3 times its normal emersion regime.

Hong Kong shores experience a strongly seasonal climate, dictated by the local monsoons. Winter is cool and dry (mean air temperature $15^{\circ} \mathrm{C}$, sea temperature $17^{\circ} \mathrm{C}$ ), whereas summer is hot and wet (mean air temperature $28^{\circ} \mathrm{C}$, maximum $36^{\circ} \mathrm{C}$, sea temperature $27^{\circ} \mathrm{C}$, see Kaehler \& Williams 1996 for details of climate). In summer, tides are lower than in winter (by $\sim 0.5 \mathrm{~m}$, maximum tidal range $\sim 2.5 \mathrm{~m}$ ) and fall during the afternoon when rock temperatures can exceed $50^{\circ} \mathrm{C}$ (Williams 1994). This combination of factors results in extremely stressful conditions for intertidal organisms and mass mortalities can occur during calm, spring low tides (Williams 1994, Williams \& Morritt 1995, Williams \& McMahon 1998, Chan \& Williams 2003). The limpet Cellana grata Gould 1859 is a mobile herbivore found between 1.5 and $2.0 \mathrm{~m}$ above Chart Datum (CD) on semi-exposed to exposed Hong Kong shores. It forages whilst awash, moving up the shore with the flooding tide and then down the shore with the ebbing tide. It becomes inactive during the ensuing low tide period in a variety of habitats (e.g. vertical surfaces, at rock-pool interfaces) that appear to alleviate thermal stress (see Williams \& Morritt 1995). If animals do not settle in such habitats, they may suffer thermal stress and, if the stress is prolonged, they often lift their shells from the rock surface showing a behaviour described as shelllifting or 'mushrooming' (Segal \& Dehnel 1962, Lewis 1963, Wolcott 1973, Garrity 1984, Williams \& Morritt 1995, Williams \& McMahon 1998).

This mushrooming behaviour in response to thermal stress has been recorded in a number of limpet species (see references above) and has been assumed to reduce heat stress by evaporative cooling as well as by minimising contact with the rock surface (Segal \& Dehnel 1962, Vermeij 1971, Verderber et al. 1983). Under extreme high-temperature stress, the limpets extend the mushrooming behaviour by lifting their shells higher off the rock surface until the foot appears distended and this may culminate in the animals being unable to maintain adhesion to the rock surface (Verderber et al. 1983, Williams \& McMahon 1998). Williams \& Morritt (1995) interpreted this as a final response to severe stress where the need for cooling may overcome the physiological problems associated with desiccation, although Wolcott (1973) previously argued that water conservation was the most important consideration under conditions of heat stress.

Ecophysiological studies performed on Cellana grata under thermal stress have shown that individuals heat up and lose water (Williams \& Morritt 1995), their haemolymph becomes increasingly acidic (Williams \& McMahon 1998), and their heart rate increases with temperature, although bradycardia may occur (Chelazzi et al. 1999). Ultimately, animals may die if such conditions do not ameliorate (i.e. the incoming tide) and this is usually preceded by the animals showing mushrooming behaviour. The physiological benefits of this behaviour remain poorly understood and are rarely related to local shore conditions. This paper examines the physiological state (body temperature, water content, heart rate and osmotic concentrations of the body fluids and mantle water) of animals performing mushrooming behaviour, observed naturally on the shore and experimentally induced in the laboratory. The responses of experimentally stressed individuals, that were either allowed to mushroom or were prevented from mushrooming, were examined to identify the possible physiological effects, and hence possible adaptive benefits, of this behaviour.

\section{MATERIALS AND METHODS}

Measurements of thermal stress on the shore. Environmental temperatures, body temperatures and heart rates of Cellana grata were measured on the shore at Cape d'Aguilar Marine Reserve (Cape d'Aguilar, Hong Kong, $22^{\circ} \mathrm{N}, 114^{\circ} \mathrm{E}$ ) in the afternoon, during a summer low water spring tide (considered a stressful period, Williams \& Morritt 1995) on 21 June 2001. Individual limpets were selected in the sequence they were encountered along a defined path and care was taken not to measure any animals that were visibly disturbed by the presence of the recorders. The approximate tidal height $( \pm 0.25 \mathrm{~m}$ above $\mathrm{CD})$ of the limpets was recorded with reference to fixed tidal height 
markers (see Williams 1993) and notes made of the habitat type occupied (approximate slope of rock, shaded or unshaded). Air temperatures $\left( \pm 0.1^{\circ} \mathrm{C}\right)$ were measured by holding a K-Type thermocouple (Omega) $\sim 2 \mathrm{~cm}$ above the rock surface and within a $2 \mathrm{~cm}$ radius of the animal. Rock surface temperatures were measured by holding the thermocouple to the rock surface within $1 \mathrm{~cm}$ of the limpet (see Williams \& Morritt 1995 for further details of temperature measurements and habitat definitions).

As limpets often lift their shells from the rock surface (= mushrooming), this height was measured using vernier calipers $( \pm 0.1 \mathrm{~mm})$. After this, the temperature of the foot was measured by inserting the thermocouple onto the foot at the rock surface and, where possible, the upper body temperature measured by inserting the thermocouple high into the apex of the shell. Heart rate (beats $\mathrm{s}^{-1}$ ) was monitored using the non-invasive method developed by Depledge \& Andersen (1990). An infrared sensor was fixed to the shell directly above the heart with Blue-Tac (Bostick) to ensure the alignment between the heart and the sensor was not disturbed by wind and light reflection. After $\sim 5 \mathrm{~min}$ (which is sufficient for the heart rate to stabilise following sensor attachment, Santini et al. 2000), the infrared reflection of the heart activity was amplified, filtered and the signal obtained visualised and recorded on a Portable Oscilloscope (Fluke 192, see Chelazzi et al. 1999 for further details). The mean value of 3 records was used as an estimate of heart rate. At the end of this procedure, the shell length of the animal was measured (vernier calipers $\pm 0.1 \mathrm{~mm}$ ). Animals were numbered with indelible ink and attempts were made to locate these individuals on the subsequent daytime low tide to determine potential loss rates.

Laboratory measurement of thermal stress. Limpets were collected from southerly facing shores at Cape d'Aguilar. Two size ranges, representing animals approaching 1 yr old (2000 cohort) and animals in their second year (the 1999 cohort), designated small and large animals (25 to 29 and 34 to $39.5 \mathrm{~mm}$ respectively, see Williams \& Morritt 1995), were collected. To minimize damage, animals were collected by hand at the end of their normal foraging period whilst still awash on the ebbing tide. Individuals were immediately placed on the lids of Petri dishes (diameter $=55 \mathrm{~mm}$, lip depth $=8 \mathrm{~mm}$ ) and transported to the Swire Institute of Marine Science (SWIMS, a distance of $\sim 400 \mathrm{~m}$ ). In the aquarium at SWIMS, animals in their individual Petri dishes were placed on an inclined surface and washed with seawater $\left(\sim 25^{\circ} \mathrm{C}\right.$ and $25 \%$ ) for $30 \mathrm{~min}$. This phase was defined as awash, and allowed animals to replenish mantle water lost during collection and to settle onto the Petri dishes. After this period, the wet weights of the animals (+ dishes) were recorded $( \pm 0.001 \mathrm{~g})$. Six randomly selected animals in each size class were removed and the osmotic concentration (= osmolality, $\operatorname{mosM~kg}{ }^{-1}$ ) of the water retained in the mantle cavity (subsequently referred to as mantle water) and the haemolymph were measured for baseline estimations.

Limpets were carefully removed from the Petri dishes in order to avoid damage to the foot. Mantle water was sampled from the nuchal cavity immediately above the head of the animal using a siliconised glass capillary. The remaining excess mantle water was then carefully removed and discarded. Once the mantle cavity was clear of excess water, the haemolymph sample was obtained by direct puncture of the pallial vein. All mantle water and haemolymph samples were temporarily stored over ice in Eppendorf microcentrifuge tubes prior to the measurement of $10 \mu$ samples using a vapour pressure osmometer (Wescor 5520, Wescor). The osmometer was regularly calibrated against Wescor 290 and 1000 mosM kg-1 $\mathrm{NaCl}$ standards.

The experimental protocol adopted was set up to simulate thermal stress conditions recorded on the shore during the same period as the field survey. Animals in each size class were transferred to an oven with air temperature at $35^{\circ} \mathrm{C}$ and $60 \%$ humidity for 60 min to simulate the drying period as the tide recedes when animals would normally become inactive on the shore. After this period, the wet weights of the animals were recorded $( \pm 0.001 \mathrm{~g})$ and 3 animals in each size class were removed, and the osmolality of mantle water and haemolymph measured as described above. These individuals were not used in any further experiments. Three limpets in each size range were then randomly assigned to treatments, either with a wire mesh cage $(5 \times 5 \mathrm{~mm}$ mesh size) attached to the Petri dish and just touching the limpets' shells (= constrained) or with a wire mesh attached to the dish but raised $\sim 10$ to $15 \mathrm{~mm}$ above the animals' shells (= unconstrained). The wire mesh in the constrained set of animals prevented the limpets from raising their shells and therefore mushrooming, whereas the unconstrained animals were able to perform this behaviour. Due to limitations in the size of the hot plate and the handling capacity for processing the measurements, experiments were repeated twice for each size group, using different individuals, to achieve 6 replicates $(n=6)$ for each treatment.

Animals in their Petri dishes were then placed on a hot plate maintained at a surface temperature of $40^{\circ} \mathrm{C}$ and an air temperature of $35^{\circ} \mathrm{C}$ for $60 \mathrm{~min}$ to simulate stressful conditions on the shore (see field data and also Williams \& Morritt 1995, Williams \& McMahon 1998). After $60 \mathrm{~min}$, the heart rate (beats $\mathrm{s}^{-1}$ ) of each individual was measured using the non-invasive 
method described above but, in the controlled laboratory environment, this was achieved without touching the animal by suspending the infrared sensor $\sim 5 \mathrm{~mm}$ above the animal's shell, directly above the heart. The wire mesh was then removed and the height that the shell was raised off the dish surface measured $( \pm 0.1 \mathrm{~mm})$. Foot temperature was then recorded $\left( \pm 0.1^{\circ} \mathrm{C}\right)$ and mantle water samples taken where possible. Animals were then wet-weighed (minus mantle water), haemolymph samples taken and shell length noted. All individuals were then dried to constant weight at $70^{\circ} \mathrm{C}$ to establish overall, water content and hence, percentage loss at different times.

A final experiment was performed to investigate responses to more extreme stress. Large limpets, which had not previously been used in experiments, were allowed to mushroom under the same experimental procedure as above, except that they were kept on the hot plate for a period of $120 \mathrm{~min}$. The responses of these animals were compared with those of the large limpets that were allowed to mushroom and were maintained on the hot plate for $60 \mathrm{~min}$.

For the shore data, analysis of covariance (ANCOVA) was employed to compare heart rate and foot temperature between mushrooming and non-mushrooming animals, using shell length as a covariate. For the laboratory experiments, potential differences between treatments were tested using analysis of variance (ANOVA) with size-class and treatment as fixed, orthogonal effects. In some cases, sample sizes were unbalanced as some measurements were not possible (e.g. mantle water). Where this occurred, General Linear Models (GLMs) were used. Assumptions of homogeneity of variances were tested using Cochran's test (for ANOVA) and Levene's test (for GLMs). As treatments were repeated for different size groups, ANOVAs were run using time as a random factor to test for a possible temporal effect. As this temporal effect proved insignificant, to increase power, the data were pooled in subsequent tests and did not include time as a factor. Two sample Student's $t$-tests were used to compare (1) recaptured and not recaptured limpets and (2) mushrooming and non-mushrooming groups from the field measurements; and (3) for the laboratory experiments, for differences between large and small limpets after initial transfer from the awash and oven phases of the experiment, and to compare large limpets maintained on the hot plate for 60 and $120 \mathrm{~min}$. Where data had unequal variances (tested using an $F$-test), the Student's $t$-test which does not require homogenous variances was used.

\section{RESULTS}

\section{Shore measurements of thermal stress}

Rock temperatures reached a maximum of $40.9^{\circ} \mathrm{C}$ and limpet body temperatures $43.5^{\circ} \mathrm{C}$ (mean values are shown in Table 1). Of the 25 limpets sampled, $76 \%$ showed a mushrooming response (Table 1) with an average height of $4.2 \pm 1.5 \mathrm{~mm} \mathrm{SD}(\max .=8.4 \mathrm{~mm})$. Although there was no difference in limpet size with tidal level ( $t$-test, $t=1.13, \mathrm{df}=17, \mathrm{p}=0.273$ ), the height of mushrooming was related to tidal level; limpets $25 \mathrm{~cm}$ lower on the shore (and thus subjected to shorter emersion times) had lower elevations $(2.14 \pm 1.89 \mathrm{~mm}$, $\mathrm{n}=14)$ than those higher on the shore $(4.54 \pm 1.98 \mathrm{~mm}$, $\mathrm{n}=11 ; t=3.081, \mathrm{df}=23, \mathrm{p}=0.005)$. Heart rate, foot and body temperatures, and air and rock temperatures, however, did not vary with tidal height (t-tests, $p>0.05$ ). Foot temperatures did not vary with size or with whether animals were mushrooming or not (ANCOVA, $\mathrm{p}>0.05$ ). Heart rate, however, interacted with limpet size (ANCOVA, $F=6.22, \mathrm{df}=1,21$, $p=0.021$ ); decreasing with size and being fastest in small, mushrooming animals. Heart rate was also higher in mushrooming than non-mushrooming limpets (ANCOVA, significant difference between slopes, $F=3.549, \mathrm{df}=2,21, \mathrm{p}=0.047) ;$ however, as the sample

Table 1. Cellana grata. Shore measurements for 25 limpets divided into those either firmly attached (= non-mushrooming) or lifting their shells from the rock surface (= mushrooming); and those that were recaptured or not found on the subsequent tide $24 \mathrm{~h}$ later

\begin{tabular}{|c|c|c|c|c|}
\hline \multirow{2}{*}{ Variable } & \multicolumn{2}{|c|}{ Mushrooming } & \multicolumn{2}{|c|}{ Recapture } \\
\hline & $\begin{array}{l}\text { Non-mushrooming } \\
\quad(\mathrm{n}=6 \pm \mathrm{SD})\end{array}$ & $\begin{array}{l}\text { Mushrooming } \\
(\mathrm{n}=19 \pm \mathrm{SD})\end{array}$ & $\begin{array}{l}\text { Not recaptured } \\
\quad(\mathrm{n}=8 \pm \mathrm{SD})\end{array}$ & $\begin{array}{c}\text { Recaptured } \\
(\mathrm{n}=17 \pm \mathrm{SD})\end{array}$ \\
\hline Shell length (mm) & $33.6 \pm 3.5$ & $35.2 \pm 4.5$ & $32.3 \pm 2.9$ & $36.1 \pm 4.3$ \\
\hline Mushroom height (mm) & Not applicable & $4.2 \pm 1.5$ & $4.3 \pm 2.5$ & $2.7 \pm 2.0$ \\
\hline Rock temperature $\left({ }^{\circ} \mathrm{C}\right)$ & $32.9 \pm 2.5$ & $35.3 \pm 2.7$ & $37.3 \pm 2.1$ & $33.5 \pm 2.2$ \\
\hline Air temperature $\left({ }^{\circ} \mathrm{C}\right)$ & $32.1 \pm 2.1$ & $33.5 \pm 1.8$ & $34.8 \pm 0.6$ & $32.5 \pm 0.4$ \\
\hline Foot temperature $\left({ }^{\circ} \mathrm{C}\right)$ & $35.7 \pm 2.9$ & $37.5 \pm 2.6$ & $39.7 \pm 2.1$ & $35.9 \pm 2.1$ \\
\hline Body temperature $\left({ }^{\circ} \mathrm{C}\right)$ & Not measured & $37.6 \pm 2.3$ & $39.3 \pm 1.6$ & $36.4 \pm 1.9$ \\
\hline Heart rate (beats s ${ }^{-1}$ ) & $1.5 \pm 0.3$ & $2.1 \pm 0.9$ & $2.8 \pm 0.9$ & $1.6 \pm 0.4$ \\
\hline
\end{tabular}




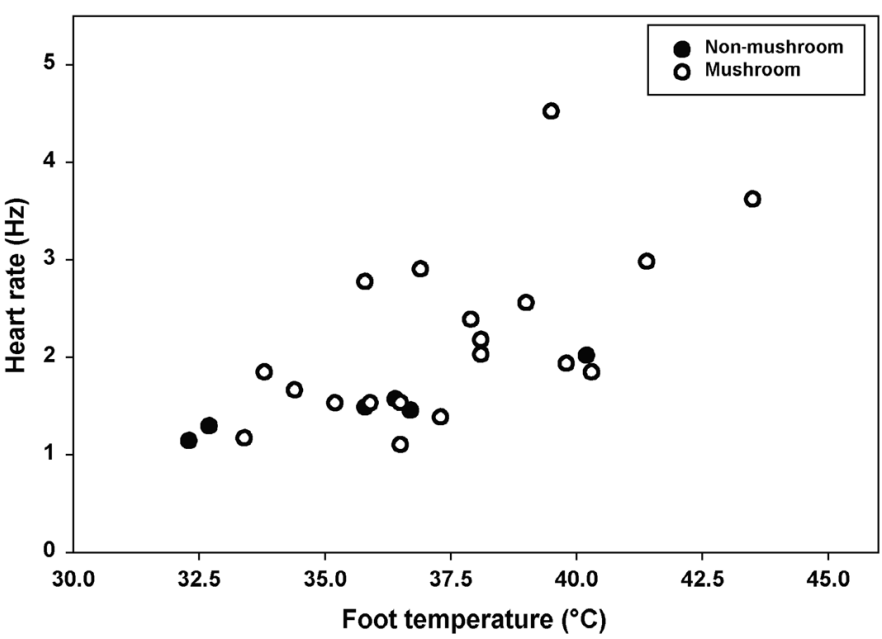

Fig. 1. Cellana grata. Relationship between foot temperatures and heart rate of mushrooming $(\mathrm{n}=19)$ and non-mushrooming $(\mathrm{n}=6)$ C. grata on the shore during a summer, low-water spring tide

size for non-mushrooming animals was small $(\mathrm{n}=6)$, this result should be viewed with caution. Heart rate was best predicted by foot temperature (linear regression, $\log$ [heart rate $]=[$ foot temperature $\times 0.0303]-$ 0.819; $F=5.696 ; \mathrm{df}=1,15, \mathrm{p}=0.031 ; \mathrm{r}^{2}=0.275$, Fig. 1), which was positively correlated with limpet body temperature, rock and air temperature (Pearson's correlations, $\mathrm{p}<0.05, \mathrm{r}=0.95,0.91$ and 0.77 for foot temperature versus body, rock and air temperature, respectively).

Of the 25 limpets measured, 8 animals (32\%) were not relocated the following day, and it is assumed that these animals had either died or been washed from the rock surface. The missing limpets were significantly smaller and had hotter foot and body temperatures and faster heart rates than animals that were recaptured the following day (Table 1, $t$-tests: $t=-2.55, \mathrm{p}=0.02, \mathrm{df}$ $=19 ; t=4.22, \mathrm{p}=0.001, \mathrm{df}=14 ; t=3.36, \mathrm{p}=0.005$, $\mathrm{df}=14$ for shell length, foot and body temperatures, respectively). Most of the limpets (6 out of 8 ) that were not recaptured had been located on horizontal rock surfaces where environmental conditions were also more severe. Air and rock temperatures were significantly hotter $(t$-tests: $t=3.17, \mathrm{p}=0.009, \mathrm{df}=11 \mathrm{df}$; $t=4.28, \mathrm{p}=0.001$, $\mathrm{df}=14$ for air and rock temperatures, respectively) than in habitats (e.g. vertical surfaces) occupied by limpets that were relocated on the next day. The majority of limpets that were not recaptured were also mushrooming ( 7 of the 8 ) and, in general, the height of mushrooming was greater in these animals than in those that survived until the next day (Table 1), although this difference was not significant ( $t$-test, p > 0.05).

\section{Laboratory measurement of thermal stress}

The experimental protocol adopted was designed to resemble the thermal stress conditions recorded on the shore during the same period as the field survey. Both the small and large limpets removed from the shore, placed on Petri dishes and washed with seawater for $30 \mathrm{~min}$ in the aquarium, replenished their mantle water (authors' pers. obs.). Both large and small animals had similar haemolymph osmolalities (Fig. 2) after the awash phase and after being in the oven for 60 min ( $t$-tests, $\mathrm{p}>0.05$ ). Limpets undergoing experimental treatments on the hot plate had more concentrated haemolymph ( $22 \%$ higher) than those which were awash or held in the oven. Small animals held on the hot plate had significantly more concentrated haemolymph than large animals (Table 2) as did constrained limpets (i.e. limpets not able to lift their shells from the dish surface) as compared to those that were able to mushroom, but this was not significant (Fig. 2, Table 2).

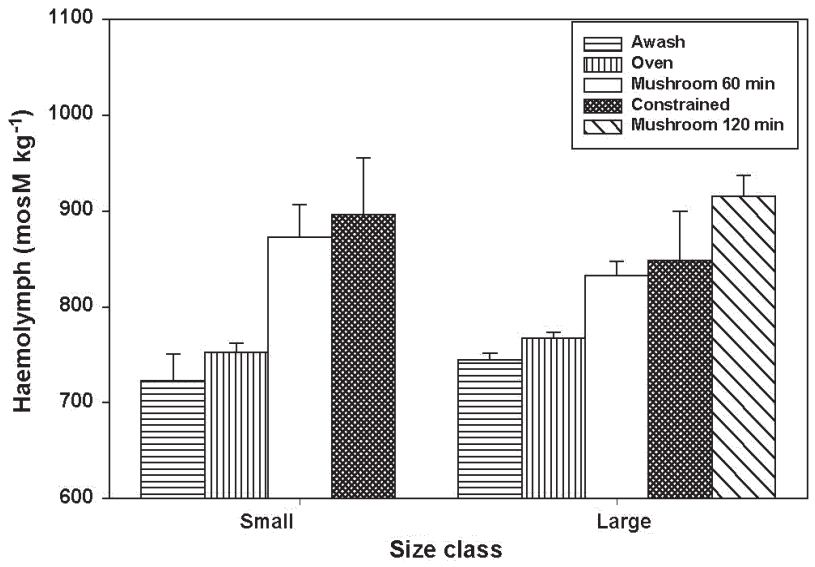

Fig. 2. Cellana grata. Mean $(+\mathrm{SD}, \mathrm{n}=6)$ haemolymph osmolalities of small and large $C$. grata either washed for $30 \mathrm{~min}$ with seawater, placed in an oven for $60 \mathrm{~min}$ or constrained and unconstrained for $60 \mathrm{~min}$ on a hot plate. Data for large $C$. grata constrained on a hot plate for 120 min are also shown

Table 2. Cellana grata. Two-way ANOVA to investigate variation in haemolymph osmolality (mosM kg${ }^{-1}$ ) in small and large C. grata prevented (= constrained) or allowed to mushroom under conditions of heat stress $(n=6)$. Variances were homogenous, Cochran's test, $C=0.47$. SNK tests were used to further separate significant factors

\begin{tabular}{|lrrrrl|}
\hline Source & df & \multicolumn{1}{c|}{ MS } & $F$ & p & SNK \\
\hline Treatment & 1 & 2262.04 & 1.81 & 0.289 & \\
Size class & 1 & 11484.37 & 6.01 & $<0.024$ & small $>$ large \\
Treat $\times$ Size & 3 & 100.04 & 0.05 & 0.821 & \\
Residual & 20 & 1910.48 & & & \\
\hline
\end{tabular}




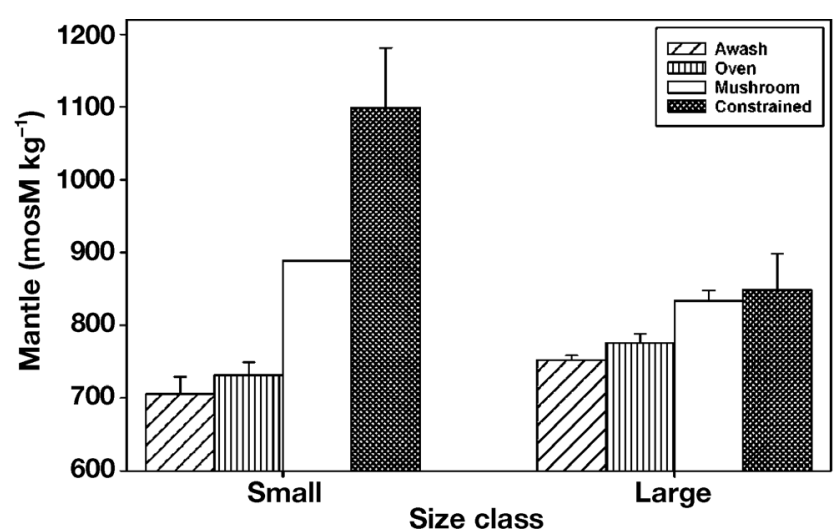

Fig. 3. Cellana grata. Mean $(+\mathrm{SD}, \mathrm{n}=1$ to 6$)$ mantle osmolalities of small and large C. grata either washed for $30 \mathrm{~min}$ with seawater, placed in an oven for 60 min or constrained and unconstrained for $60 \mathrm{~min}$ on a hot plate. Note, for small, unconstrained animals mantle water could only be collected from 1 individual

Large animals, in contrast, had more concentrated mantle water concentrations than small animals after both the awash (Fig. 3, $t=-4.88, \mathrm{df}=10, \mathrm{p}=0.001$ ) and oven phase (Fig. $3, t=-3.75, \mathrm{df}=7, \mathrm{p}=0.007$ ). It proved very difficult to collect mantle water from small, unconstrained, mushrooming animals after they had spent time on the hot plate (Fig. 3). Mantle water was, however, collected from some small, constrained limpets, and this was much more concentrated ( $24 \%$ higher) than for large animals (Fig. 3, Table 3). In the case of large limpets, it was possible to collect mantle water from most experimental animals and there was no difference between constrained or unconstrained animals. Mantle water osmolality of animals held on the hot plate was, however, consistently higher than those rehydrated or maintained in the oven for 60 min (Fig. 3). In general, mantle water and haemolymph osmolalities across all treatments showed a strong positive correlation (Fig. 4; Pearson's correlation, $\mathrm{r}=0.917, \mathrm{p}<0.001$, $\mathrm{n}=37$ ), although in small constrained limpets, mantle

Table 3. Cellana grata. One-way GLM to investigate variation in mantle water osmolality (mosM kg-1) in small and large C. grata prevented (= constrained) and allowed to mushroom under conditions of heat stress ( $\mathrm{n}=3$ to 6 ). Note that the design did not include small animals which were allowed to mushroom as mantle water could only be obtained from 1 individual. Variances were homogenous, Levene's test $=1.17$. SNK tests were used to further separate significant factors

\begin{tabular}{lcrcc|} 
Source & df & MS & $F$ & $p$ \\
\hline Treatment & 2 & 80333 & 35.31 & $<0.0001$ \\
Residual & 12 & 2275 & & \\
SNK tests: & & & & \\
Small, constrained & $>$ large, constrained & large, mushroom \\
\hline
\end{tabular}

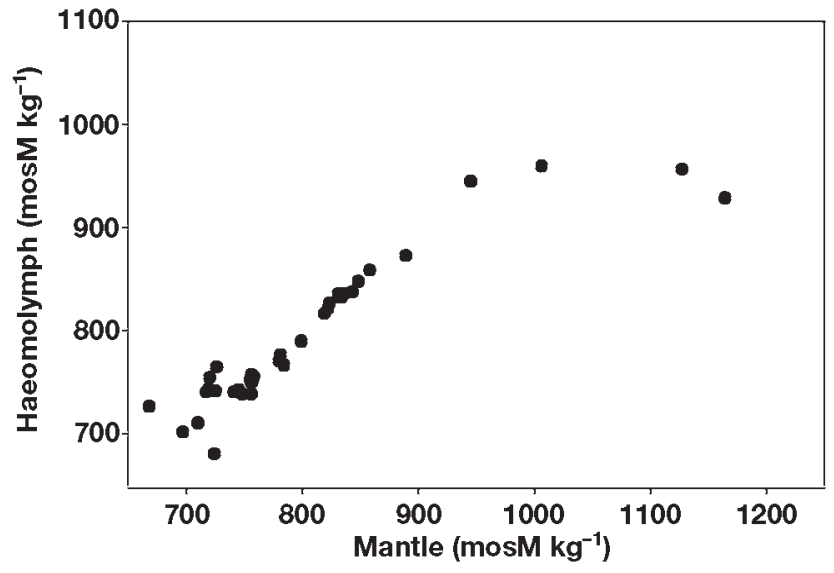

Fig. 4. Cellana grata. Relationship between haemolymph and mantle osmolality pooled for all experimental treatments. Points >1000 mosM kg-1 for mantle osmolality are all small, constrained limpets

water concentration was much more concentrated than haemolymph concentration (Figs. 2 \& 3).

Limpets that were unconstrained showed mushrooming behaviour, with large animals raising their shells higher than small animals (small $=3.25 \pm 0.49 \mathrm{~mm}$ $\mathrm{SD}$, large $=4.47 \pm 1.0 \mathrm{~mm}, t$-test: $t=2.66, \mathrm{df}=7$, $\mathrm{p}=0.033$ ), whereas animals that were constrained could only lift their shells by $\leq 1 \mathrm{~mm}$. When scaled for body size, however, there was no difference between the size classes in the relative height of mushrooming ( $t$-test, $\mathrm{p}>0.05$ ) as mushrooming height was positively, but weakly, related to shell size (Pearson's correlation, $r=0.668, p=0.018, n=12$ ). Foot temperatures also showed a treatment effect that was not related to size

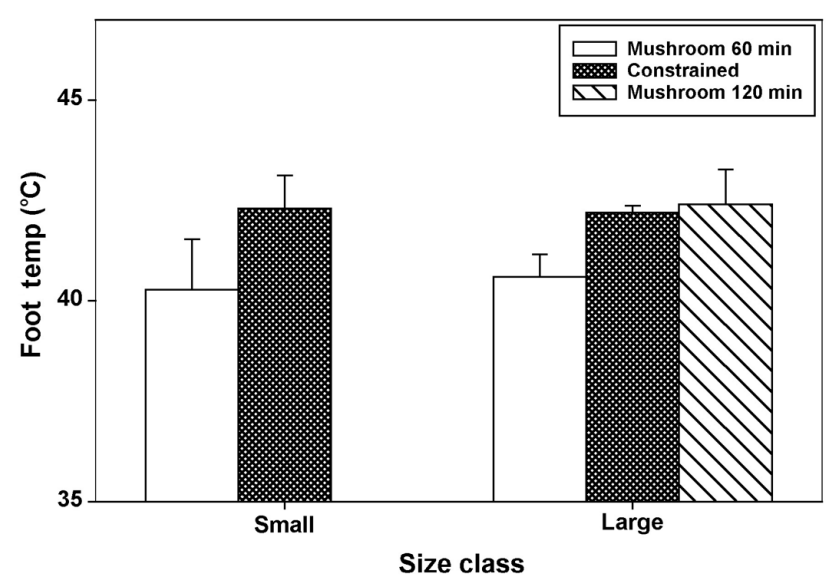

Fig. 5. Cellana grata. Mean (+SD) foot temperatures for small and large constrained and unconstrained C. grata after 60 min on a hot plate. Data for large C. grata constrained on a hot plate for $120 \mathrm{~min}$ are also shown 
class (Fig. 5, Table 4). Constrained animals had higher $\left(\sim 2^{\circ} \mathrm{C}\right)$ foot temperatures than those that were able to mushroom, irrespective of size. Heart rates of constrained limpets were, however, slower than those allowed to mushroom (Fig. 6, Table 5) and heart rates of small limpets were greater than those of large animals. There was, however, no interaction between these treatments. Small limpets consistently lost a higher percentage of their body water than large animals, both after $60 \mathrm{~min}$ in the oven $(t$-test: $t=-3.98$,

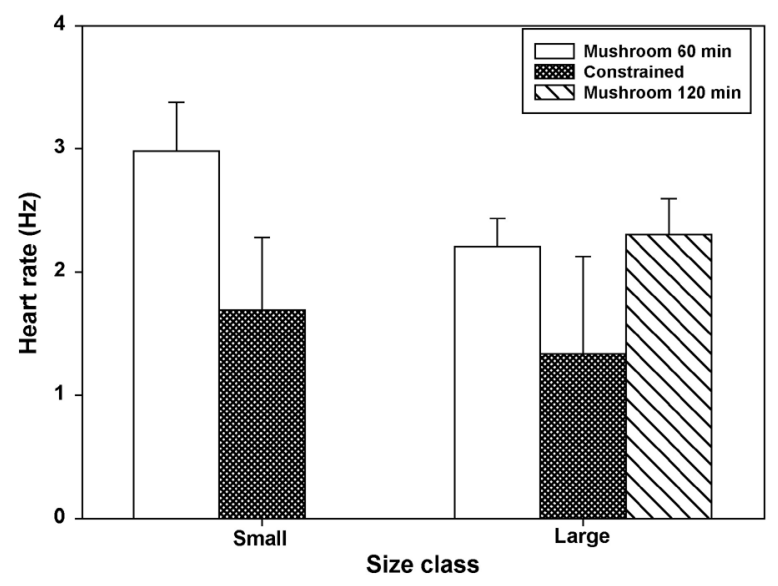

Fig. 6. Cellana grata. Mean $(+\mathrm{SD}, \mathrm{n}=6)$ heart rates for small and large constrained and unconstrained $C$. grata after 60 min on a hot plate. Data for large C. grata constrained on a hot plate for $120 \mathrm{~min}$ are also shown

Table 4. Cellana grata. Two-way GLM to investigate variation in foot temperature $\left({ }^{\circ} \mathrm{C}\right)$ in small and large $C$. grata prevented and allowed to mushroom under conditions of heat stress ( $n=3$ to 6). Variances were homogenous, Levene's test $=1.62$. SNK tests were used to further separate significant factors

\begin{tabular}{|lrlccl|}
\hline Source & df & MS & $F$ & $p$ & SNK \\
\hline Treatment & 1 & 13.08 & 15.3 & 0.002 & mushroom $>$ constrained \\
Size class & 1 & 0.046 & 0.05 & 0.818 & \\
Treat $\times$ Size & 1 & 0.1736 & 0.20 & 0.659 & \\
Residual & 14 & 0.855 & & & \\
\hline
\end{tabular}

Table 5. Cellana grata. Two-way GLM to investigate variation in heart rate (beats $\mathrm{s}^{-1}$ ) in small and large C. grata prevented and allowed to mushroom under conditions of heat stress $(n=5$ to 6$)$. Variances were homogenous, Levene's test $=0.35$. SNK tests were used to further separate significant factors

\begin{tabular}{|lrrrrl|}
\hline Source & df & MS & \multicolumn{1}{c|}{$F$} & p & SNK \\
\hline Treatment & 1 & 6.65 & 21.15 & $<0.0001$ & mushroom $>$ constrained \\
Size class & 1 & 1.82 & 5.78 & 0.027 & small $>$ large \\
Treat $\times$ Size & 1 & 0.25 & 0.80 & 0.382 & \\
Residual & 20 & 0.31 & & & \\
\hline
\end{tabular}

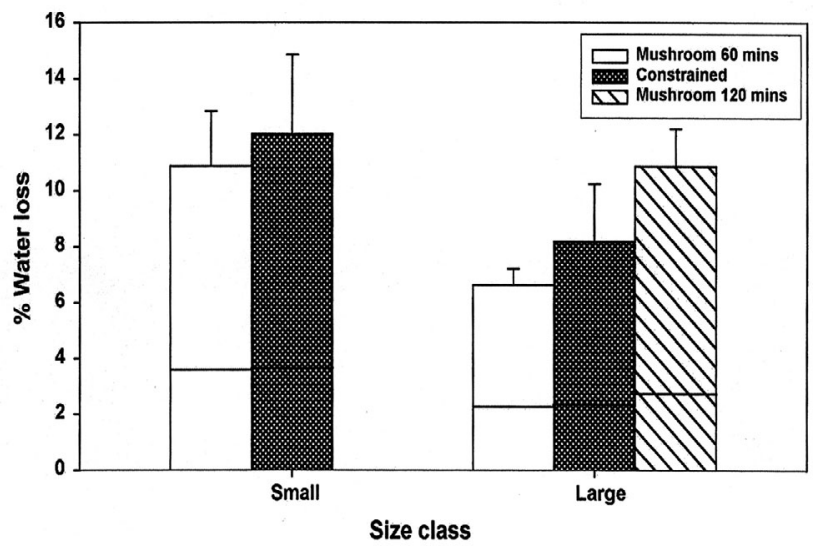

Fig. 7. Cellana grata. Mean $(+\mathrm{SD}, \mathrm{n}=6)$ percentage water loss for small and large constrained and unconstrained C. grata after $60 \mathrm{~min}$ in an oven (below horizontal line) and $60 \mathrm{~min}$ on a hot plate (above the horizontal line). Data for large C. grata constrained on a hot plate for $120 \mathrm{~min}$ are also shown

$\mathrm{df}=14, \mathrm{p}=0.001)$ and after $60 \mathrm{~min}$ on the hot plate (Fig. 7, Tables 6 \& 7). Constrained limpets lost more water than those allowed to mushroom on the hot plate (Table 6), but this was not significant for total water loss (over the whole period of 120 min of oven plus hotplate treatment), and there was also no significant interaction between treatment and size in terms of total water loss (Table 7).

Large limpets, that were allowed to mushroom but maintained on the hot plate for $120 \mathrm{~min}$, showed simi-

Table 6. Cellana grata. Two-way ANOVA to investigate the variation in percent water loss in small and large C. grata prevented and allowed to mushroom under conditions of heat stress after $60 \mathrm{~min}$ on a hot plate $(\mathrm{n}=6)$. Variances were homogenous, Cochran's test, $C=0.47$. SNK tests were used to further separate significant factors

\begin{tabular}{|lrrrrl|}
\hline Source & df & \multicolumn{1}{c}{ MS } & \multicolumn{1}{c|}{$F$} & p & \multicolumn{1}{c|}{ SNK } \\
\hline Treatment & 1 & 10.15 & 5.11 & 0.035 & constrained $>$ mushroom \\
Size class & 1 & 43.55 & 21.94 & 0.0001 & small $>$ large \\
Treat $\times$ Size & 1 & 0.24 & 0.12 & 0.73 & \\
Residual & 20 & 2.152 & & & \\
\hline
\end{tabular}

Table 7. Cellana grata. Two-way ANOVA to investigate the variation in total percent water loss in small and large $C$. grata prevented and allowed to mushroom $(\mathrm{n}=6)$. Variances were homogenous, Cochran's test, $C=0.56$. SNK tests were used to further separate significant factors

\begin{tabular}{|lrrrcc|}
\hline Source & df & MS & \multicolumn{1}{c}{$F$} & $\mathrm{p}$ & SNK \\
\hline Treatment & 1 & 10.76 & 3.02 & 0.098 & \\
Size class & 1 & 97.81 & 27.43 & $<0.0001$ & small > large \\
Treat $\times$ Size & 1 & 0.242 & 0.07 & 0.797 & \\
Residual & 20 & 3.565 & & & \\
\hline
\end{tabular}


lar mushroom heights and heart rates to those maintained on the hot plate for $60 \mathrm{~min}$ ( $t$-test: $\mathrm{p}>0.05$, Fig. 6). Foot temperatures were, however, higher ( $t$-test: $t=-3.21, \mathrm{df}=10, \mathrm{p}=0.015$, Fig. 5) and these limpets lost nearly twice as much water whilst on the hot plate compared with animals held for $60 \mathrm{~min}$ ( $t$-test, unequal variances: $t=6.86, \mathrm{df}=7, \mathrm{p}<0.001$, Fig. 7) although similar loss rates were recorded in the oven (t-test: $\mathrm{p}>0.05$, Fig. 7). It was not possible to sample mantle water from any of the limpets maintained on the hot plate for $120 \mathrm{~min}$, but the haemolymph osmolality of these limpets was also much higher than in limpets maintained for $60 \mathrm{~min}$ on the hot plate (t-test: $t=-7.57, \mathrm{df}=10, \mathrm{p}<0.001$, Fig. 2)

In general, therefore, limpets of both size classes experienced a similar thermal regime on the hot plate in terms of foot temperature. Unconstrained limpets were able to mushroom, and larger animals were able to lift their shells further from the surface than small individuals. Constrained animals, which were unable to mushroom, had lower heart rates, but higher foot temperatures and haemolymph osmolalities and, in the case of small animals, higher mantle water osmolality. Small animals lost a higher proportion of water, and also had higher haemolymph concentrations and heart rates than large animals. Small animals also lost mantle water on the hot plate, especially if they were able to mushroom. Large animals kept for longer periods of time on the hot plate showed similar mushroom heights and heart rates as those maintained for a shorter period, but had higher foot temperatures, lost all their mantle water and nearly twice as much of their total water content, and had much higher haemolymph osmolality.

\section{DISCUSSION}

During low tide periods at Cape d'Aguilar, Hong Kong, the limpet Cellana grata experiences heat and desiccation stress. Animals that rest in unprotected habitats (horizontal rock surfaces, west-facing slopes) can experience high rock temperatures $\left(>50^{\circ} \mathrm{C}\right.$, Williams \& Morritt 1995) and can be exposed for $\sim 6 \mathrm{~h}$ to potentially stressful low-tide periods with extended periods of insolation. In the present study, temperatures were not extreme (max. rock temperature $>40^{\circ} \mathrm{C}$ ) although maximum limpet body temperatures reached $43.5^{\circ} \mathrm{C}$. It is not unusual for animal temperatures to be higher than the rock temperature (Lewis 1963, Vermeij 1971, Williams \& Morritt 1995), probably as a result of a reduction in cooling of the limpet foot, which will be directly heated by insolation and conduction from the bed rock, as compared to the open rock surface, which will be cooled by air move- ment. Limpet foot temperature was related to rock, air and limpet body temperatures, and was a good predictor of heart rate. Chelazzi et al. (1999) have shown that the heart rate of $C$. grata increases with temperature over the range 28 to $40^{\circ} \mathrm{C}$. Above this temperature, however, this relationship is not always linear and intermittent bradycardia is sometimes observed, which Chelazzi et al. $(1999,2001)$ have argued is a means by which $C$. grata may attempt to regulate body temperature and reduce metabolism.

Even under these relatively mild stressful conditions, most animals showed a mushrooming response, raising their shells from the rock surface. Animals that did not show this response were found in cooler habitats (vertical and shaded surfaces), and had lower foot temperatures and lower heart rates, suggesting they were not as stressed as those animals that did mushroom. Limpets that did mushroom had elevated foot temperatures and heart rates, and were found in habitats with higher rock temperatures than those that did not mushroom, although this relationship was not significant, possibly due to the small sample size for animals that did not lift their shells. Limpets higher on the shore (by $\sim 25 \mathrm{~cm}$ ), and therefore emersed for longer time periods, had greater mushroom heights than those lower on the shore, suggesting a clear effect of duration of aerial exposure as well as of overall temperature effects on this behaviour.

A $68 \%$ recapture rate was achieved on the day after the field survey. Limpets not recaptured may have died, or been lost to the observers. Recapture of non-stressed animals is usually $>85 \%$ (Williams \& Morritt 1995) and so it seems likely that a large proportion of those not recaptured did die. Periodic mass mortalities of Cellana grata do occur during calm, spring low tides (Williams \& Morritt 1995) and mortality rates vary greatly depending on the specific day to day conditions (A. Ngan unpubl. data). Retrospective comparison of records for the lost animals with those which were recaptured, showed that these limpets were smaller, had higher mushrooming heights, higher foot and body temperatures, and in particular higher heart rates compared to those that were recaptured. This suggests that these animals were experiencing more severe stress than those which survived and were recaptured the next day, and may account for the elevated loss rate. The shore measurements, therefore, suggest a relationship between thermal stress and mushrooming, which varies with duration of exposure (tidal height) and thermal regime experienced (micro-habitat). This relationship will determine, on a given day, the relative survival of limpets on the shore (Williams \& Morritt 1995) as also proposed for mussels (Helmuth \& Hofmann 2001). 


\section{What is the benefit of mushrooming?}

The laboratory experiments attempted to mimic sublethal thermal stress and were conducted at the same time of day as limpets would normally experience these conditions on the shore so as to control for any potential endogenous rhythm. Animals were collected whilst awash, prior to their inactive phase when they would be stationary on the shore and exposed to stressful emersion conditions. Prior to placement on the hot plates, different-sized limpets rehydrated by the awash phase in the aquarium and then dried in the oven, showed similar haemolymph osmolalities. Large animals, however, had more concentrated mantle water osmolalities after both the awash and the oven phase, although the reasons for this difference are unclear.

After the desiccation period of $60 \mathrm{~min}$ in the oven at $35^{\circ} \mathrm{C}$ and while subsequently being held on the hot plate $\left(40^{\circ} \mathrm{C}\right)$ for either 60 or $120 \mathrm{~min}$, unconstrained animals exhibited mushrooming behaviour. The height of the shell lifted from the surface was greater for large than for small limpets, although the relative height was proportional to size. The constrained animals could only lift their shells very slightly. There were a variety of different responses shown by the large and small animals under these 2 treatments. In general, constrained animals had higher foot temperatures and haemolymph osmolalities, but lower heart rates than limpets allowed to mushroom. Overall, water loss was greater in small animals, much of this loss being from the mantle water.

After $60 \mathrm{~min}$ on the hot plate, small animals that were able to mushroom had lost their mantle water and this could only be sampled from 1 animal. Loss of extra-corporal water (= mantle water) after $1.5 \mathrm{~h}$ was also recorded for small Collisella pelta (Lowell 1984). Mantle water osmolality was more concentrated in small animals that were unable to mushroom than in the single mushrooming individual. Mantle water could be sampled from the large limpets, but not from those allowed to mushroom on the hot plate for 120 min. Mantle water osmolality was greater in small, constrained limpets than in large animals, which showed similar concentrations between those mushrooming and those constrained. Haemolymph samples could, however, be collected from all animals. This is in contrast to the work of Segal \& Dehnel (1962) who were unable to collect blood (= haemolymph) samples from severely desiccated limpets (Acmaea) but could still collect extra-visceral fluids (mantle water). Their techniques were, however, different to the ones employed in this study, which may account for this difference. Constrained limpets had slightly higher haemolymph concentrations than those allowed to mushroom, and the concentrations in small animals were significantly more concentrated than in large animals. Large limpets allowed to mushroom and maintained on a hot plate for $120 \mathrm{~min}$ also had higher haemolymph concentrations than individuals maintained for only $60 \mathrm{~min}$.

As the limpets experienced progressive stress, they lost their mantle water or it became increasingly concentrated. This would result in the gills drying up and impede gas and metabolite exchange via the mantle cavity (see Williams \& McMahon 1998). Haemolymph concentrations also showed a similar trend. Presumably, whilst mantle water is available (i.e. not lost via evaporation), it can be used to exchange respiratory waste products but will also become more concentrated as water evaporates (see the 1 example of the small limpets where a sample was obtained). Constrained animals were better able to maintain their mantle water but the osmolality became increasingly higher, presumably as exchange between body fluids and mantle water was maintained.

There was a linear, positive, relationship between haemolymph and mantle osmolality, as recorded for Acmaea (Segal \& Dehnel 1962). This relationship held true until haemolymph osmolalities reached 950 mosM kg-1 when haemolymph values stabilised, despite increasing mantle water concentrations, although this is based on relatively few observations of small constrained individuals. This suggests some active maintenance of haemolymph values below a certain threshold, or the accelerated concentration of ions in water in the mantle cavity as this fluid evaporates. A dynamic equilibrium has been proposed between mantle and limpet body fluids, resulting in differential passage of solutes depending on the osmotic concentration of these 2 fluid reservoirs (Segal \& Dehnel 1962, Williams \& Morritt 1995). The maintenance of a 'water jacket' of mantle water has been suggested to help minimize dehydration and the subsequent concentration of body fluids (Segal \& Dehnel 1962). As this water is lost, however, body fluids will start to evaporate (cooling the animal) and haemolymph osmolality increases, as has been described in the highshore littorinid Cenchritis muricatus during extended desiccation stress (Emson et al. 2002). The current results for $C$. grata, however, suggest that under severe stress, there may be some evidence for short-term regulation of the haemolymph relative to the mantle water. Whilst such osmoregulation is unusual in molluscs, there are some instances where this may occur during stressful periods, e.g. aestivating Pomacea spp. (Little 1968).

An increase in body fluid osmolality has been recorded in numerous limpet species with increasing desiccation (Segal \& Dehnel 1962, Wolcott 1973, 
Branch 1981, Gallien 1985) and has been associated with impedance of $\mathrm{CO}_{2}$ excretion and increasing acidification of the haemolymph (Marshall \& McQuaid 1992, Williams \& McMahon 1998). Variation in ionic balance is likely to be extremely important, as haemolymph has been shown to acidify and in some cases become hypermagnesic (Patella depressa Chaisermartin, 1971), resulting in loss of adhesion, and limpets subsequently being swept away by waves. Wolcott (1973) suggested that it was concentration of the body fluids that ultimately caused mortality in Acmaea species, as water loss, in his study, rarely reached lethal limits. This may be true for Acmaea species which Wolcott recorded as tolerating high degrees of water loss (>70\%, recorded in laboratory conditions) and onshore thermal conditions that were never observed to exceed the limpets' tolerance levels over a 3 yr period. In Cellana grata, however, tolerance of high osmotic concentrations is unlikely to be as great, as mortality can occur within an emersion period after only $30 \%$ loss of water (Williams \& Morritt 1995).

Water loss was also greater in small than in large limpets, and in large animals allowed to mushroom and kept on the hot plate for $120 \mathrm{~min}$. Higher water loss rate in small limpets is a common effect of desiccation stress (Collins 1979, Lowell 1984, Williams \& Morritt 1995), presumably as a function of surface area: volume ratio (Branch 1975). Limpets allowed to mushroom also lost less water after $60 \mathrm{~min}$ on the hot plate compared to those that were constrained. This appears counterintuitive, as mushrooming has always been assumed to be a response to stress that allows evaporative cooling, reducing heat stress but at the expense of desiccation risk (Segal \& Dehnel 1962, Vermeij 1971, Garrity 1984). The present study does not agree with this supposition as constrained animals actually lost more water than those allowed to mushroom. This suggests the loss of overall water may be associated with their higher temperatures. Large animals that were stressed for $2 \mathrm{~h}$ lost a similar amount of water per hour as those stressed for $1 \mathrm{~h}(\sim 6.7 \%$ loss in the first hour versus $11.2 \%$ after $2 \mathrm{~h}$ ), similar to the total loss for small limpets after $1 \mathrm{~h}$. Extended emersion clearly leads to an increase in physical stress with these animals losing twice as much water. At this rate, the lethal level ( 30\%, Williams \& Morritt 1995) would be achieved after $\sim 5 \mathrm{~h}$, which would represent an extremely stressful, midday spring low-tide period. Wolcott (1973) observed lifting of the shell at high temperatures but not under desiccating conditions, proposing that this behaviour was evidence of impending heat coma.

Foot temperatures of the 2 size classes maintained for $1 \mathrm{~h}$ on the hot plate were similar $\left(\sim 40^{\circ} \mathrm{C}\right)$ although constrained animals had higher foot temperatures than those allowed to mushroom. Being able to mushroom, therefore, permits limpets to cool their foot temperatures by $\sim 2^{\circ} \mathrm{C}$. A cooler foot temperature has also been shown for Scurria stipulata and Siphonaria gigas permitted to lift their shells as compared to experimental animals constrained by tapping their shells with a pencil and forcing them to clamp onto the substrate (Garrity 1984). Whilst these animals lost more water than those that remained clamped to the surface, they also suffered reduced mortality (although this was not significant in the case of Siphonaria), again supporting an adaptive function for this behaviour.

Heart rate has been shown to be positively related to foot temperature (Chelazzi et al. 1999) and is assumed to be indicative of metabolic activity (Marshall \& McQuaid 1992, Santini et al. 1999). In this case, however, mushrooming limpets had higher heart rates than the constrained animals and small animals had higher rates than large animals. This suggests that mushrooming of the foot and lifting the shell is an active process, incurring metabolic cost. This may also indicate constant active circulation in an attempt to cool the haemolymph, or to increase gaseous and ionic exchange at the gills whilst the animals are experiencing the first stage of heat stress. Chelazzi et al. (1999) proposed that limpets should decrease circulation under conditions of severe heat stress to reduce such circulation (which would heat the haemolymph at the site of the gills) to maintain a cooler, inner body temperature, and such a response was recorded for animals under increased desiccation stress (Chelazzi et al. 2001). The foot temperatures of limpets maintained on the hot plate for $120 \mathrm{~min}$ were also higher $\left(\sim 2^{\circ} \mathrm{C}\right)$ than those maintained for $60 \mathrm{~min}$. In this case, the positive relationship between foot temperature and heart rate appears decoupled as has been suggested by Chelazzi et al. (1999) under conditions of increased heat stress and limpets may be attempting to lower their heart rate to minimise metabolic activity, as a means of regulating internal body temperature (Chelazzi et al. 1999, 2001).

\section{Mushrooming behaviour as a short-term gamble?}

Limpets on the shore, when stressed, exhibit mushrooming behaviour. This behaviour is related to foot temperatures, which are controlled by environmental conditions, especially as a result of habitat choice. Small animals and those in stressful habitats (horizontal rock surfaces, high on the shore) exhibit prolonged mushrooming and can die. Mushrooming appears to be an active process, involving increased heart rates. This behaviour cools the limpets, probably due to decreasing heat conduction from the rock and increas- 
ing heat loss by convection with the air. There is no evidence that this behaviour enhances evaporative cooling, as there was no difference in overall water loss in mushrooming animals compared to those limpets which could not lift their shells. Loss of mantle water is, however, more rapid and may play a cooling role, but also results in subsequent physiological impacts on the animals (lack of gaseous exchange, haemolymph concentration and acidification) and this may be associated with increased circulation rates. This suggests evaporative cooling does not affect body fluids initially and it is only after longer durations ( $>2 \mathrm{~h}$ ) of emersion that this occurs. Brief periods ( 1 or $2 \mathrm{~h}$ ) of mushrooming, therefore, appear to have short-term positive benefits and it is only under very stressful conditions or prolonged emersion that the consequences of this response become fatal, especially for smaller animals. Mushrooming behaviour is, therefore, proposed to be a short-term gamble, involving an energetic cost which allows limpets temporary physiological relief from stressful conditions and perhaps increases their chance of surviving until the next tidal immersion.

Acknowledgements. Many thanks for great field assistance from Shirley, Chow Chi Yui, and Janet, Lee Ka Wai, and discussions with Roger Uglow. C. Law supplied excellent technical assistance and together with $\mathrm{L}$. $\mathrm{Ng}$ helped prepare the figures. Permission to work in the Cape d'Aguilar Marine Reserve was granted by the Agriculture, Fisheries and Conservation Department, Hong Kong SAR Government. K.M.Y.L. was supported by the Croucher Foundation, Hong Kong and D.M. acknowledges financial support from The Royal Society (UK). K.M.Y.L., D.M. and M.D.P. are grateful to the Swire Institute of Marine Science and Department of Ecology \& Biodiversity, The University of Hong Kong for providing accommodation and research facilities. Thanks to Prof G. Chelazzi (Firenze, Italy) and the Hard Rock Ecology Group (HKU) for helpful comments on the manuscript.

\section{LITERATURE CITED}

Branch GM (1975) The ecology of Patella from the Cape Peninsula, South Africa. IV. Desiccation. Mar Biol 32: 179-188

Branch GM (1981) The biology of limpets: physical factors, energy flow, and ecological interactions. Oceanogr Mar Biol Annu Rev 19:235-380

Chaisemartin C (1970) Le 'stress' hydrominéral et la zonation des Patellidae. CR Séanc Soc Biol Filiales 164:2088-2091

Chan BKK, Williams GA (2003) The impact of physical stress and molluscan grazing on the settlement and recruitment of Tetraclita species (Cirripedia: Balanomorpha) on a tropical shore. J Exp Mar Biol Ecol 284:1-23

Chelazzi G, Williams GA, Gray DG (1999) Field and laboratory measurement of heart rate in a tropical limpet, Cellana grata. J Mar Biol Assoc UK 79:749-751

Chelazzi G, De Pirro M, Williams GA (2001) Cardiac responses to abiotic factors in two tropical limpets, occurring at different tidal levels of the shore. Mar Biol 139: 1079-1085

Collins LS (1979) Abundance, substrate angle, and desicca- tion resistance in two sympatric species of limpets. Veliger 192:199-203

Connell JH (1961) Effects of competition, predation by Thais lapillus, and other factors on natural populations of the barnacle Balanus balanoides. Ecol Monogr 31:61-104

Depledge MH, Anderson BB (1990) A computer-aided physiological monitoring system for continuous, long-term recording of cardiac activity in selected invertebrates. Comp Biochem Phys A 96:474-477

Emson, RH, Morritt D, Andrews EB, Young CM (2002) Life on a hot dry beach: behavioural, physiological, and ultrastructural adaptations of the littorinid gastropod Cenchritis (Tectarius) muricatus. Mar Biol 140:723-732

Foster BA (1971) On the determinants of the upper limit of intertidal distribution of barnacles (Crustacea: Cirripedia). J Anim Ecol 40:33-48

Frank PW (1965) The biodemography of an intertidal snail population. Ecology 46:831-844

Gallien WB (1985) The effects of aggregations on water loss in Collisella digitalis. Veliger 28:14-17

Garrity SD (1984) Some adaptations of gastropods to physical stress on a tropical rocky shore. Ecology 65:559-574

Hawkins SJ, Hartnoll RG (1985) Factors determining the upper limits of intertidal canopy-forming algae. Mar Ecol Prog Ser 20:265-271

Helmuth BST, Hofmann GE (2001) Microhabitats, thermal heterogeneity, and patterns of physiological stress in the rocky intertidal zone. Biol Bull (Woods Hole) 201:374-384

Kaehler S, Williams GA (1996) Distribution of algae on tropical rocky shores: spatial and temporal patterns of non-coralline encrusting algae in Hong Kong. Mar Biol 125:177-187

Kohn AJ (1993) Episodic mortality of limpets on a shore platform at Rottnest Island, Western Australia. In: Wells F, Walker D, Kirkman H, Letterbridge R (eds) Fifth international marine biology workshop: the marine flora and fauna of Rottnest Island, Western Australia. Western Australia Museum, Perth, p 497-508

Lewis JB (1963) Environmental and tissue temperatures of some tropical intertidal marine animals. Biol Bull (Woods Hole) 124:277-284

Little C (1968) Aestivation and ionic regulation in two species of Pomacea (Gastropoda; Prosobranchia). J Exp Biol 48: $569-585$

Lowell RB (1984) Desiccation of intertidal limpets: effects of shell size, fit to substratum, and shape. J Exp Mar Biol Ecol 77:197-207

Marshall DJ, McQuaid CD (1992) Comparative aerial metabolism and water relations of the intertidal limpets Patella granularis L. (Mollusca: Prosobranchia) and Siphonaria oculus Kr. (Mollusca: Pulmonata). Physiol Zool 65:1040-1056

McMahon BR, Burggren WW, Pinder AW, Wheatly MG (1991) Air exposure and physiological compensation in a tropical intertidal chiton, Chiton stokesii (Mollusca: Polyplacophora). Physiol Zool 64:728-747

McMahon RF (2001) Acute thermal tolerance in intertidal gastropods relative to latitude, superfamily, zonation and habitat with special emphasis on the littorinidae. J Shellfish Res 20:459-467

Santini G, Pirro MD, Chelazzi G (1999) In situ and laboratory assessment of heart rate in a Mediterranean limpet using a noninvasive technique. Physiol Biochem Zool 72:198-204

Santini G, Williams GA, Chelazzi G (2000) Assessment of factors affecting heart rate of the limpet Patella vulgata on the natural shore. Mar Biol 137:291-296

Segal E, Dehnel PA (1962) Osmotic behaviour in an intertidal limpet, Acmaea limatula. Biol Bull (Woods Hole) 122: $417-430$ 
Smith SL (1975) Physiological ecology of the limpet Cellana ornata (Dillwyn). NZ J Mar Freshw Res 9:395-402

Underwood AJ (1985) Physical factors and biological interactions: the necessity and nature of ecological experiments. In: Moore PG, Seed R (eds) The ecology of rocky coasts. Hodder \& Stoughton, London, p 372-390

Verderber GW, Cook SB, Cook CB (1983) The role of the home scar in reducing water loss during aerial exposure of the pulmonate limpet, Siphonaria alternata (Say). Veliger 25:235-243

Vermeij GJ (1971) Temperature relationships of some tropical Pacific intertidal gastropods. Mar Biol 10:308-314

Williams GA (1993) Seasonal variation in algal species richness and abundance in the presence of molluscan herbivores on a tropical rocky shore. J Exp Mar Biol Ecol 167: 261-275

Editorial responsibility: Otto Kinne (Editor-in-Chief), Oldendorf/Luhe, Germany
Williams GA (1994) The relationship between shade and molluscan grazing in structuring communities on a moderately-exposed tropical rocky shore. J Exp Mar Biol Ecol 178:79-95

Williams GA, McMahon BR (1998) Haemolymph pH and oxygen levels in a naturally stressed tropical limpet, Cellana grata. In: Morton BS (ed) The third international conference on the marine biology of the South China Sea, Hong Kong. Hong Kong University Press, Hong Kong, p 239-245

Williams GA, Morritt D (1995) Habitat partitioning and thermal tolerance in a tropical limpet, Cellana grata. Mar Ecol Prog Ser 124:89-103

Wolcott TG (1973) Physiological ecology and intertidal zonation in limpets (Acmaea): a critical look at 'limiting factors'. Biol Bull (Woods Hole) 145:389-422

Submitted: April 27, 2004; Accepted: November 30, 2004 Proofs received from author(s): April 19, 2005 